\title{
A SHORT NOTE ON CONTAINMENT OF CORES
}

\author{
Kyungyong Lee \\ Department of Mathematics, University of Michigan, Ann Arbor, Michigan, \\ USA \\ kyungl@umich.edu
}

\begin{abstract}
We show that cores of ideals do not preserve the inclusion.
\end{abstract}

Huneke and Swanson 2 raised the question of whether, given integrally closed ideals $I \subset I^{\prime}$ in a ring $R$, it is necessarily true that $\operatorname{core}(I) \subset \operatorname{core}\left(I^{\prime}\right)$. Hyry and Smith [4, Corollary 5.5.1] gave a partial answer. The purpose of this note is to show that the answer is no in general. Although the simplest counterexample is a principal ideal $I$ which is contained in a power $I^{\prime}$ of a maximal ideal, we are more interested in the case when both $I$ and $I^{\prime}$ have the same height.

Let $R=k[x, y, z, w]_{(x, y, z, w)}$ with $k$ a field of characteristic zero and let $\mathfrak{m}$ denote the maximal ideal of $R$. Let $I=I_{2}+\mathfrak{m}^{3}$, where $I_{2}=\left(x^{2}+y w, y^{2}+z w, z^{2}+\right.$ $x w)$. The computer algebra system Macaulay shows that $I_{2}$ is radical. Then it is not hard to check that $I$ is integrally closed (see 7, Lemma 2.1]). It follows from [8] or [3] that core $(I)=J^{n+1}: I^{n}$, where $J=\left(x^{2}+y w, y^{2}+z w, z^{2}+x w, w^{3}\right)$ and $n$ is the least integer such that $I^{n+1}=J I^{n}$. In fact $J$ is a minimal reduction of $I$ and the computer algebra system Macaulay shows $I^{2}=J I$.

On the other hand, if we take $I^{\prime}=\mathfrak{m}^{2}$ so that $I \subset I^{\prime}$, then $\operatorname{core}\left(I^{\prime}\right)=$ $\operatorname{core}\left(\mathfrak{m}^{2}\right)=\mathfrak{m}^{5}$ by [5, Theorem 1.3] or [1, Proposition 4.2]. But the computer algebra system Macaulay shows that $\operatorname{core}(I)=J^{2}: I=I^{2} \not \subset \mathfrak{m}^{5}$, therefore $\operatorname{core}(I) \not \subset \operatorname{core}\left(I^{\prime}\right)$. This gives a negative answer to the above question.

More generally, we have the following conjecture.

Conjecture 1. Let $R=k\left[x_{1}, \ldots, x_{n}\right]_{\left(x_{1}, \ldots, x_{n}\right)}$ with $k$ a field of characteristic zero and let $\mathfrak{m}$ denote the maximal ideal of $R$. Let $I=I_{d}+\mathfrak{m}^{d+1}$, where $I_{d}$ is a complete intersection ideal of $s$ general $d$-forms $(1 \leq s<n)$. Let $b=\left\lfloor\frac{d n-s+1}{d+1}\right\rfloor$ and $a=d n-s+1-(d+1) b$. Then

$$
\operatorname{core}(I)=\mathfrak{m}^{a} I^{b} .
$$

Theorem 2. The conjecture holds true for $d=1$.

Proof. Let $X=\operatorname{Spec} R$. By [4, Corollary 5.3.1], we have

$$
\operatorname{core}(I)=\mathcal{J}(X, n \cdot I) \text {. }
$$

To get the simplest log resolution $\mu: X_{2} \rightarrow X_{1} \rightarrow X$ of $(X, I)$, we blow up $X$ at the origin, and then blow up the resulting surface $X_{1}$ along the intersection 
of the exceptional divisor, say $E_{1}$, and the proper transform of the linear space defined by $I_{1}$. Let $E_{2} \subset X_{2}$ denote the second exceptional divisor. Then, using the notations in [6, Definition 9.2.3], we get

$$
K_{X_{2} / X}=(n-1) E_{1}+(n+s-1) E_{2}, \quad I \cdot \mathcal{O}_{X_{2}}=\mathcal{O}_{X_{2}}\left(-E_{1}-2 E_{2}\right),
$$

so

$$
\begin{aligned}
\mathcal{J}(X, n \cdot I) & =\mu_{*} \mathcal{O}_{X_{2}}\left((n-1) E_{1}+(n+s-1) E_{2}-n\left(E_{1}+2 E_{2}\right)\right) \\
& =\mu_{*} \mathcal{O}_{X_{2}}\left(-E_{1}-(n-s+1) E_{2}\right) \\
& =\mathfrak{m}^{n-s+1-2\left\lfloor\frac{n-s+1}{2}\right\rfloor} I^{\left\lfloor\frac{n-s+1}{2}\right\rfloor}
\end{aligned}
$$

Remark 3. In the situation of the above conjecture, if $d \geq 2$ then core $(I)$ and $\mathcal{J}(n \cdot I)$ do not agree in general. But the above example leads to the following question.

If we define the deeper-core of $I \subset R$ by

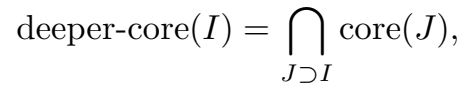

then is

$$
\operatorname{deeper-\operatorname {core}}(I)=\mathcal{J}(\operatorname{Spec} R, n \cdot I)
$$

always true?

The author is grateful to Craig Huneke, Rob Lazarsfeld, Karen Smith and Irena Swanson for valuable discussions and correspondence. He would like to thank the referee for the helpful suggestion.

\section{References}

[1] Corso, A., Polini, C., Ulrich, B. (2002), Core and residual intersections of ideals. Trans. Amer. Math. Soc. 354:2579-2594.

[2] Huneke, C., Swanson, I. (1995), Cores of ideals in 2-dimensional regular local rings, Michigan Math. J. 42:193-208.

[3] Huneke, C., Trung, N. V.(2005), On the core of ideals. Compos. Math. 141:1-18.

[4] Hyry, E., Smith, K. E. (2003), On a Non-vanishing Conjecture of Kawamata and the Core of an Ideal, Amer. J. Math. 125:1349-1410.

[5] Hyry, E., Smith, K. E. (2004), Core versus graded core, and global section of line bundles, Trans. Amer. Math. Soc. 356:3143-3166. 
[6] Lazarsfeld, R. (2004), Positivity in Algebraic Geometry II. volume 49 of Ergebnisse der Mathematik und ihrer Grenzgebiete. 3. Folge. A Series of Modern Survey in Mathematics [Results in Mathematics and Related Areas. 3rd Series. A Series of Modern Survey in Mathematics]. Springer-Verlag, Berlin. Positivity in vector bundles, and multiplier ideals.

[7] Lazarsfeld, R., Lee, K. (2007), Local Syzygies of Multiplier ideals, Invent. Math. 167:409-418.

[8] Polini, C., Ulrich, B. (2005), A formula for the core of an ideal. Math. Ann. 331:487-503. 